\title{
Spontaneous CP Violation in a 2HDM with Controlled Scalar FCNC
}

\section{Miguel Nebot*}

${ }^{a}$ Departamento de Física and Centro de Física Teórica de Partículas (CFTP), Instituto Superior Técnico (IST), U. de Lisboa (UL),

Av. Rovisco Pais 1, P-1049-001 Lisboa, Portugal.

E-mail: miguel.r.nebot.gomez@tecnico.ulisboa.pt

A viable minimal model with spontaneous $\mathrm{CP}$ violation in the framework of Two Higgs Doublet Models is proposed. The model is based on a generalised Branco-Grimus-Lavoura model with a flavoured $\mathbb{Z}_{2}$ symmetry, under which two of the quark families are even and the third one is odd. The lagrangian respects $\mathrm{CP}$ invariance, but the vacuum has a $\mathrm{CP}$ violating phase, which is able to generate a complex CKM matrix, with the strength of CP violation compatible with experiment. In this model, the presence of scalar mediated flavour changing neutral couplings is deeply connected to the generation of a complex CKM matrix from the vacuum phase. Concerning the scalar sector, the most relevant aspect is that the new scalar particles are necessarily lighter than $1 \mathrm{TeV}$. A complete analysis of the model including the most relevant constraints is performed, showing that it is viable and that it has definite implications for the observation of New Physics signals in, for example, flavour changing Higgs decays or the discovery of the new scalars at the LHC. We give special emphasis to processes like $t \rightarrow \mathrm{h} c, \mathrm{~h} u$, as well as $\mathrm{h} \rightarrow b s, b d$, which are relevant for the LHC and the ILC.

European Physical Society Conference on High Energy Physics - EPS-HEP2019 -

10-17 July, 2019

Ghent, Belgium

\footnotetext{
* Speaker.
} 


\section{A 2HDM with SCPV and Scalar FCNC}

We refer the interested reader to [1] for further details on the motivations for the study of Spontaneous CP Violation in Two Higgs Doublet models, originally introduced by T.D. Lee in [2]. Concerning controlled FCNC, Branco-Grimus-Lavoura models - which have SFCNC controlled by the CKM matrix itself - were introduced in [3], and their phenomenology explored in [4, 5]; more general symmetry controlled scenarios have been explored in $[6,7]$.

\subsection{Scalar Sector}

We consider a $2 \mathrm{HDM}$ with symmetry under the $\mathbb{Z}_{2}$ transformation $\Phi_{1} \mapsto \Phi_{1}, \Phi_{2} \mapsto-\Phi_{2}$, softly broken by $\Phi_{1}^{\dagger} \Phi_{2}, \Phi_{2}^{\dagger} \Phi_{1}$ terms. In addition, we impose CP invariance of the lagrangian. These two requirements are satisfied by the scalar potential

$$
\begin{aligned}
\mathscr{V}\left(\Phi_{1}, \Phi_{2}\right)=\mu_{11}^{2} & \Phi_{1}^{\dagger} \Phi_{1}+\mu_{22}^{2} \Phi_{2}^{\dagger} \Phi_{2}+\mu_{12}^{2}\left(\Phi_{1}^{\dagger} \Phi_{2}+\Phi_{2}^{\dagger} \Phi_{1}\right)+\lambda_{1}\left(\Phi_{1}^{\dagger} \Phi_{1}\right)^{2}+\lambda_{2}\left(\Phi_{2}^{\dagger} \Phi_{2}\right)^{2} \\
& +2 \lambda_{3}\left(\Phi_{1}^{\dagger} \Phi_{1}\right)\left(\Phi_{2}^{\dagger} \Phi_{2}\right)+2 \lambda_{4}\left(\Phi_{1}^{\dagger} \Phi_{2}\right)\left(\Phi_{2}^{\dagger} \Phi_{1}\right)+\lambda_{5}\left[\left(\Phi_{1}^{\dagger} \Phi_{2}\right)^{2}+\left(\Phi_{2}^{\dagger} \Phi_{1}\right)^{2}\right],
\end{aligned}
$$

with all parameters real. Electroweak symmetry is spontaneously broken by the vacuum expectation values

$$
\left\langle\Phi_{1}\right\rangle=\left(\begin{array}{c}
0 \\
e^{i \theta_{1}} v_{1} / \sqrt{2}
\end{array}\right), \quad\left\langle\Phi_{2}\right\rangle=\left(\begin{array}{c}
0 \\
e^{i \theta_{2}} v_{2} / \sqrt{2}
\end{array}\right) .
$$

As usual, we use $\theta=\theta_{2}-\theta_{1}, v^{2}=v_{1}^{2}+v_{2}^{2}, c_{\beta}=\cos \beta \equiv v_{1} / v, s_{\beta}=\sin \beta \equiv v_{2} / v$ and $t_{\beta} \equiv \tan \beta$, with $v_{1} \geq 0, v_{2} \geq 0$. From the minimization conditions one obtains $\cos \theta=\frac{-\mu_{12}^{2}}{2 \lambda_{5} v_{1} v_{2}}$; furthermore, one can trade $\mu_{11}^{2}, \mu_{22}^{2}$ and $\mu_{12}^{2}$ for other parameters and choose $v_{1}^{2}+v_{2}^{2}=v^{2}=(246 \mathrm{GeV})^{2}$ for appropriate electroweak symmetry breaking without loss of generality. One is left with a candidate minimum characterised by the values of $\theta$ and $\tan \beta=v_{2} / v_{1}$, which, together with the remaining free parameters in the scalar sector, atre subject to requirements such as boundedness from below of the scalar potential or perturbative unitarity bounds on high energy scattering of scalars. In a "Higgs basis" $\left\{H_{1}, H_{2}\right\}$ only $H_{1}$ acquires a vacuum expectation value

$$
\left\langle H_{1}\right\rangle=\frac{v}{\sqrt{2}}\left(\begin{array}{l}
0 \\
1
\end{array}\right), \quad\left\langle H_{2}\right\rangle=\left(\begin{array}{l}
0 \\
0
\end{array}\right) .
$$

Expansion of the fields around the vacuum gives the physical scalars h, H, A (neutral) and $\mathrm{H}^{ \pm}$; it is assumed that $\mathrm{h}$ is the lightest scalar, the $125 \mathrm{GeV}$ "Higgs-like" scalar. Identification of the physical neutral scalars involves an orthogonal $3 \times 3$ mixing matrix $\mathscr{R}$, relevant for the final form of the Yukawa couplings of the quarks (see [1] for details).

\subsection{Yukawa couplings and Flavour Structure}

The flavour structure of the model is defined by requiring invariance of the Yukawa lagrangian

$$
\mathscr{L}_{\mathrm{Y}}=-\bar{Q}_{L}^{0}\left(\Gamma_{1} \Phi_{1}+\Gamma_{2} \Phi_{2}\right) d_{R}^{0}-\bar{Q}_{L}^{0}\left(\Delta_{1} \tilde{\Phi}_{1}+\Delta_{2} \tilde{\Phi}_{2}\right) u_{R}^{0}+\text { H.c. },
$$

under the $\mathbb{Z}_{2}$ transformations

$$
\begin{aligned}
& \Phi_{1} \mapsto \Phi_{1}, \quad \Phi_{2} \mapsto-\Phi_{2}, \quad Q_{L 3}^{0} \mapsto-Q_{L 3}^{0}, \quad Q_{L j}^{0} \mapsto Q_{L j}^{0}, \quad j=1,2, \\
& d_{R k}^{0} \mapsto d_{R k}^{0}, \quad u_{R k}^{0} \mapsto u_{R k}^{0}, \quad k=1,2,3 .
\end{aligned}
$$


Apart from $\Phi_{2}$, only one left-handed doublet, $Q_{L 3}^{0}$, has a non-trivial transformation. Invariance under eq. (1.5) imposes the following general form of the Yukawa coupling matrices:

$$
\Gamma_{1}=\left(\begin{array}{ccc}
\times & \times & \times \\
\times & \times & \times \\
0 & 0 & 0
\end{array}\right), \quad \Gamma_{2}=\left(\begin{array}{ccc}
0 & 0 & 0 \\
0 & 0 & 0 \\
\times & \times & \times
\end{array}\right), \quad \Delta_{1}=\left(\begin{array}{ccc}
\times & \times & \times \\
\times & \times & \times \\
0 & 0 & 0
\end{array}\right), \quad \Delta_{2}=\left(\begin{array}{ccc}
0 & 0 & 0 \\
0 & 0 & 0 \\
\times & \times & \times
\end{array}\right) .
$$

The symmetry assignment in eq. (1.5) and the Yukawa matrices in eq. (1.6) correspond to the generalised BGL models introduced in [6]. Furthermore, in order to impose CP invariance at the Lagrangian level, we also require the Yukawa couplings to be real $\Gamma_{j}^{*}=\Gamma_{j}, \Delta_{j}^{*}=\Delta_{j}$. In the Higgs basis,

$$
\mathscr{L}_{\mathrm{Y}}=-\frac{\sqrt{2}}{v} \bar{Q}_{L}^{0}\left(M_{d}^{0} H_{1}+N_{d}^{0} H_{2}\right) d_{R}^{0}-\frac{\sqrt{2}}{v} \bar{Q}_{L}^{0}\left(M_{u}^{0} \tilde{H}_{1}+N_{u}^{0} \tilde{H}_{2}\right) u_{R}^{0}+\text { H.c. },
$$

which allows the identification of the quark mass matrices $M_{d}^{0}, M_{u}^{0}$. The matrices in eq. (1.7) read

$$
\begin{array}{ll}
M_{d}^{0}=\frac{v e^{i \theta_{1}}}{\sqrt{2}}\left(c_{\beta} \Gamma_{1}+e^{i \theta} s_{\beta} \Gamma_{2}\right), & N_{d}^{0}=\frac{v e^{i \theta_{1}}}{\sqrt{2}}\left(-s_{\beta} \Gamma_{1}+e^{i \theta} c_{\beta} \Gamma_{2}\right), \\
M_{u}^{0}=\frac{v e^{-i \theta_{1}}}{\sqrt{2}}\left(c_{\beta} \Delta_{1}+e^{-i \theta} s_{\beta} \Delta_{2}\right), & N_{u}^{0}=\frac{v e^{-i \theta_{1}}}{\sqrt{2}}\left(-s_{\beta} \Delta_{1}+e^{-i \theta} c_{\beta} \Delta_{2}\right) .
\end{array}
$$

We set, without loss of generality, $\theta_{1}=0$ for simplicity. Following the "row structure" in eq. (1.6), we can write:

$$
M_{d}^{0}=\left(\begin{array}{ccc}
1 & 0 & 0 \\
0 & 1 & 0 \\
0 & 0 & e^{i \theta}
\end{array}\right) \hat{M}_{d}^{0}, \quad M_{u}^{0}=\left(\begin{array}{ccc}
1 & 0 & 0 \\
0 & 1 & 0 \\
0 & 0 & e^{-i \theta}
\end{array}\right) \hat{M}_{u}^{0},
$$

with $\hat{M}_{d}^{0}$ and $\hat{M}_{u}^{0}$ real. Then, the bi-diagonalisation of $M_{d}^{0}$ and $M_{u}^{0}$ gives the following CKM matrix $V \equiv \mathscr{U}_{L}^{u \dagger} \mathscr{U}_{L}^{d}$

$$
V=\mathscr{O}_{L}^{u T}\left(\begin{array}{ccc}
1 & 0 & 0 \\
0 & 1 & 0 \\
0 & 0 & e^{i 2 \theta}
\end{array}\right) \mathscr{O}_{L}^{d}, \quad \mathscr{O}_{L}^{d}, \mathscr{O}_{L}^{u} \in O(3)
$$

For generic $\mathscr{O}_{L}^{u}$ and $\mathscr{O}_{L}^{d}$, if $e^{i 2 \theta} \neq \pm 1, V$ in eq. (1.11) incorporates an irremovable source of CP violation, that is, the model has spontaneous $\mathrm{CP}$ violation as desired. The presence of Scalar FCNC is encoded in the matrices $N_{d}, N_{u}$ matrices; they have the following form

$$
\begin{aligned}
& {\left[N_{d}\right]_{i j}=t_{\beta} \delta_{i j} m_{d_{i}}-\left(t_{\beta}+t_{\beta}^{-1}\right) \hat{n}_{[\mathrm{d}] i}^{*} \hat{n}_{[\mathrm{d}] j} m_{d_{j}},} \\
& {\left[N_{u}\right]_{i j}=t_{\beta} \delta_{i j} m_{u_{i}}-\left(t_{\beta}+t_{\beta}^{-1}\right) \hat{n}_{[\mathrm{u}] i}^{*} \hat{n}_{[\mathrm{u}] j} m_{u_{j}},}
\end{aligned}
$$

where $\hat{n}_{[\mathrm{d}]}$ and $\hat{n}_{[\mathrm{u}]}$ are complex unitary vectors, not independent but related through

$$
\hat{n}_{[\mathrm{d}] i}=\hat{n}_{[\mathrm{u}] j} V_{j i}, \quad \hat{n}_{[\mathrm{u}] i}=V_{i j}^{*} \hat{n}_{[\mathrm{d}] j} .
$$

With these ingredients, the Yukawa couplings of the neutral and charged scalars are completely specified (see, again, [1] for further details). Introducing the hermitian and antihermitian combinations

$$
H_{q} \equiv \frac{N_{q}+N_{q}^{\dagger}}{2}, \quad A_{q} \equiv \frac{N_{q}-N_{q}^{\dagger}}{2}
$$


we have

$$
\begin{aligned}
\mathscr{L}_{S \overline{\mathrm{qq}}}= & -\frac{S}{v}\left\{\bar{d}\left[\mathscr{R}_{1 s} M_{d}+\mathscr{R}_{2 s} H_{d}+i \mathscr{R}_{3 s} A_{d}\right] d+\bar{d}\left[\mathscr{R}_{2 s} A_{d}+i \mathscr{R}_{3 s} H_{d}\right] \gamma_{5} d\right\} \\
& -\frac{S}{v}\left\{\bar{u}\left[\mathscr{R}_{1 s} M_{u}+\mathscr{R}_{2 s} H_{u}-i \mathscr{R}_{3 s} A_{u}\right] u+\bar{u}\left[\mathscr{R}_{2 s} A_{u}-i \mathscr{R}_{3 s} H_{u}\right] \gamma_{5} u\right\},
\end{aligned}
$$

with $s=1,2,3$ for $S=\mathrm{h}, \mathrm{H}$, A, respectively, and

$$
\mathscr{L}_{\mathrm{H}^{ \pm} \overline{\mathrm{q}} \mathrm{q}}=-\frac{\sqrt{2} \mathrm{H}^{+}}{v}\left[\bar{u}_{L} V N_{d} d_{R}-\bar{u}_{R} N_{u}^{\dagger} V d_{L}\right]-\frac{\sqrt{2} \mathrm{H}^{-}}{v}\left[\bar{d}_{R} N_{d}^{\dagger} V^{\dagger} u_{L}-\bar{d}_{L} V^{\dagger} N_{u} u_{R}\right] .
$$

With eqs. (1.12)-(1.13), $\left[H_{q}\right]_{i j}$ and $\left[A_{q}\right]_{i j}$ in eq. (1.15) read

$$
\left[H_{q}\right]_{i j}=t_{\beta} \delta_{i j} m_{q_{i}}-\left(t_{\beta}+t_{\beta}^{-1}\right) \hat{n}_{[\mathrm{q}] i}^{*} \hat{n}_{[\mathrm{q}] j} \frac{m_{q_{i}}+m_{q_{j}}}{2}, \quad\left[A_{q}\right]_{i j}=\left(t_{\beta}+t_{\beta}^{-1}\right) \hat{n}_{[\mathrm{q}]}^{*} \hat{n}_{[\mathrm{q}] j} \frac{m_{q_{i}}-m_{q_{j}}}{2} .
$$

\subsection{Phenomenology}

After the outline of the model in the previous section, a short summary of the full phenomenological study included in [1] is presented. The main constraints imposed the analysis are the following: CKM matrix: moduli $\left|V_{i j}\right|$ in the first and second rows and phase $\gamma$ in agreement with data; scalar sector verifying boundedness, perturbative unitarity, oblique parameter constraints and $m_{\mathrm{H}}$, $m_{\mathrm{A}}, m_{\mathrm{H}^{ \pm}}>150 \mathrm{GeV}$; production $\times$ decay signal strengths of the $125 \mathrm{GeV}$ Higgs-like scalar $\mathrm{h}$; neutral meson mixings; $\operatorname{Br}\left(B \rightarrow X_{s} \gamma\right)$; rare top decays $t \rightarrow \mathrm{h} q$.

The analysis has two main goals: (1) to establish that the model is viable after a reasonable set of constraints is imposed; (2) to explore the prospects for the observation of some definite non$\mathrm{SM}$ signal. We concentrate in particular on flavour changing decays $t \rightarrow \mathrm{h} c, \mathrm{~h} u$ and $\mathrm{h} \rightarrow b s, b d$, of interest, respectively, for the LHC and the ILC. We also consider a representative low energy observable, the time dependent CP violating asymmetry in $B_{s} \rightarrow J / \Psi \Phi, A_{J / \Psi \Phi}^{C P}$, for which the SM prediction is $A_{J / \Psi \Phi}^{C P} \simeq-0.04$, while current results give $-0.030 \pm 0.033$, leaving significant room for New Physics contributions. Further implications for the phenomenology of $\mathrm{H}, \mathrm{A}$ and $\mathrm{H}^{ \pm}$, in particular for the observation of these new scalars at the LHC, vary significantly between allowed regions in the parameter space of the model and can also depend on details of the lepton sector (which are not considered here), and thus we do not address these additional implications further. A selection of results is shown in Figure 1.

Figures 1(a), 1(b) and 1(c) illustrate straightforwardly the absence of a decoupling regime in this model. Figures 1(d) and 1(e) illustrate the prospects for some definite deviations with respect to SM expectations in different flavour changing neutral transitions. Figure 1(d) shows $\operatorname{Br}(\mathrm{h} \rightarrow b s) \equiv \operatorname{Br}(\mathrm{h} \rightarrow \bar{b} s+b \bar{s})$ vs. $A_{J / \Psi \Phi}^{C P}$; it is interesting to notice that: (i) $\operatorname{Br}(\mathrm{h} \rightarrow b s)$ can reach values as large as $10^{-2}$, relevant for searches at the ILC, and (ii) significant deviations of the $\mathrm{SM}$ expectation $A_{J / \Psi \Phi}^{C P} \simeq-0.036$ can arise. Finally, Figure 1 (e) shows the most relevant flavour decays involving $\mathrm{h}, \mathrm{h} \rightarrow b s$ in the down quark sector and $t \rightarrow \mathrm{h} c$ in the up quark sector: it is clear that there is sufficient available parameter space for the model to have both decays within experimental reach, although, obviously, having both rates in that interesting region is not compulsory. 


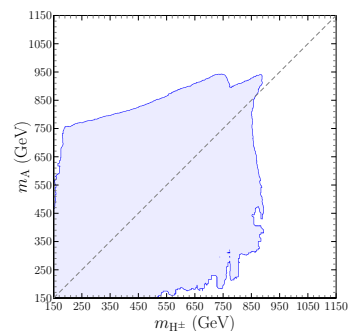

(a) $m_{\mathrm{A}}$ vs. $m_{\mathrm{H}^{ \pm}}$.

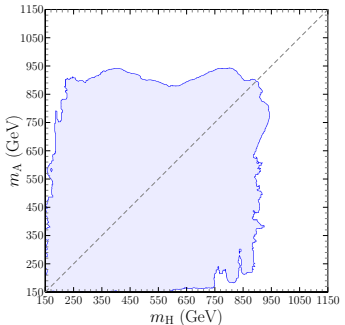

(b) $m_{\mathrm{A}}$ vs. $m_{\mathrm{H}}$.

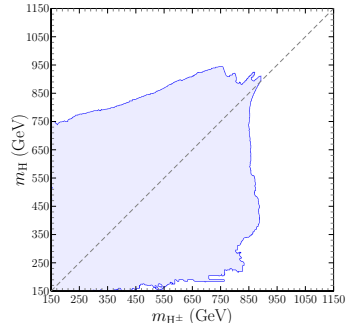

(c) $m_{\mathrm{H}}$ vs. $m_{\mathrm{H}^{ \pm}}$.

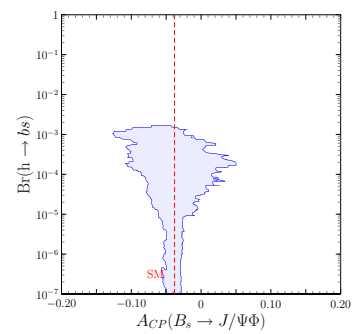

(d) $\operatorname{Br}(\mathrm{h} \rightarrow b s)$ vs. $A_{J / \Psi \Phi}^{C P}$

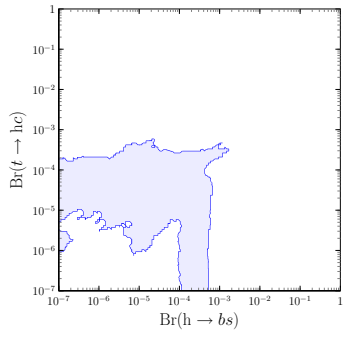

(e) $t \rightarrow \mathrm{h} c$ vs. $\mathrm{h} \rightarrow b s$.

Figure 1: Region allowed at $99 \%$ C.L. by the requirements of the full analysis.

\section{Conclusions}

A 2HDM where CP Violation has a spontaneous origin, which also generates a realistic CKM matrix, and where Scalar Flavour Changing Neutral Couplings are present with controlled intensity, has been discussed. Among the relevant phenomenological consequences of the model, the existence of new scalars with masses below $1 \mathrm{TeV}$, and the possibility of having flavour changing processes such as $t \rightarrow \mathrm{h} c$ or $\mathrm{h} \rightarrow b s$ within experimental reach, have been explored.

\section{References}

[1] M. Nebot, F. J. Botella, and G. C. Branco, Vacuum Induced CP Violation Generating a Complex CKM Matrix with Controlled Scalar FCNC, Eur. Phys. J. C79 (2019), no. 8 711, [1808. 00493 ].

[2] T. Lee, A Theory of Spontaneous T Violation, Phys.Rev. D8 (1973) 1226-1239.

[3] G. Branco, W. Grimus, and L. Lavoura, Relating the scalar flavor changing neutral couplings to the CKM matrix, Phys.Lett. B380 (1996) 119-126, [hep-ph/9601383].

[4] F. Botella, G. Branco, A. Carmona, M. Nebot, L. Pedro, and M. Rebelo, Physical Constraints on a Class of Two-Higgs Doublet Models with FCNC at tree level, JHEP 1407 (2014) 078, [1401.6147].

[5] F. J. Botella, G. C. Branco, M. Nebot, and M. N. Rebelo, Flavour Changing Higgs Couplings in a Class of Two Higgs Doublet Models, Eur. Phys. J. C76 (2016), no. 3 161, [1508 . 05101 ].

[6] J. M. Alves, F. J. Botella, G. C. Branco, F. Cornet-Gomez, and M. Nebot, Controlled Flavour Changing Neutral Couplings in Two Higgs Doublet Models, Eur. Phys. J. C77 (2017), no. 9585 , [1703.03796].

[7] J. M. Alves, F. J. Botella, G. C. Branco, F. Cornet-Gomez, M. Nebot, and J. P. Silva, Symmetry Constrained Two Higgs Doublet Models, Eur. Phys. J. C78 (2018), no. 8 630, [1803.11199]. 